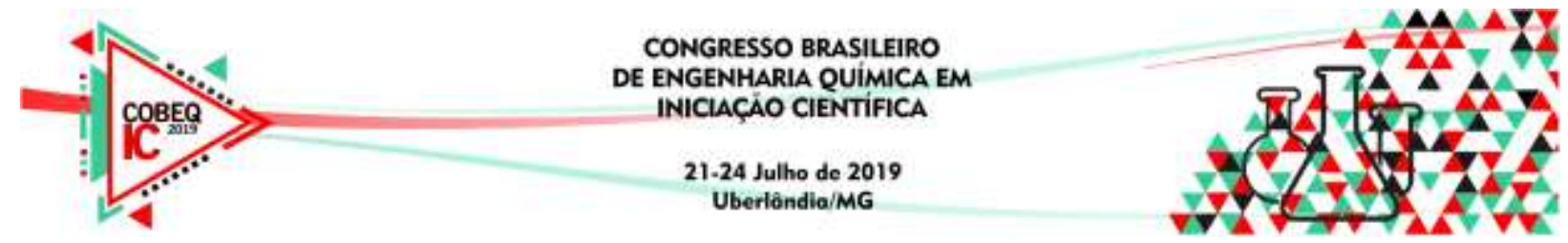

\title{
ESTUDO DA DISTRIBUIÇÃO DO TEMPO DE RESIDÊNCIA NO ESCOAMENTO ENTRE COMPARTIMENTOS DE UNIDADE DE FLOTAÇÃO
}

\author{
P. G. AGUIAR ${ }^{1}$, R. L. O. ROCHESTER ${ }^{2}$, J. C. GONÇALVES ${ }^{1}$ \\ ${ }^{1}$ Instituto Federal do Norte de Minas Gerais, Faculdade de Engenharia Química \\ ${ }^{2}$ Universidade Estadual de Montes Claros, Faculdade de Engenharia Civil \\ E-mail para contato: polyana.eq@gmail.com
}

\begin{abstract}
RESUMO - No presente trabalho, foram determinadas as Distribuições de Tempo de Residência nos segmentos que abarcam o processo de flotação por ar dissolvido: mistura rápida, zona de contato e zona de separação. Foi utilizada a técnica de perturbação-resposta com traçador colorimétrico. O comportamento do escoamento de fluido entre os compartimentos do flotador apresentou-se conforme modelos discutidos em literatura, demonstrando aplicabilidade na específica unidade de processo.
\end{abstract}

\section{INTRODUÇÃO}

Unidades de flotação podem ser estudadas como reatores de escoamento contínuo nos quais os coagulantes são adicionados na unidade de mistura rápida e os flocos retirados na zona de separação (CESTARI et al., 2012). No floculador, os flocos são formados para posterior remoção da fase líquida na etapa de flotação propriamente dita. Os compartimentos podem apresentar dois tipos de modelos de escoamento ideal, pistão e mistura completa ou mistura perfeita (LEVENSPIEL, 2000).

A Distribuição do Tempo de Residência (DTR) é fundamental na avaliação do grau de desvio da idealidade de um escoamento. Segundo Levenspiel (2000), os elementos de um fluido num recipiente podem adotar diferentes trajetórias e gastar tempos distintos para atravessá-lo.

O tempo de residência em um sistema pode ser comparado ao tempo de residência teórico, ou tempo espacial $(\tau)$, que é aquele onde não são considerados agitações variadas, desvios, imperfeições e geometrias que oferecem graus de mistura diferentes no sistema. O tempo espacial (Equação (1)) leva em conta apenas o volume e o tempo de percurso do fluido no equipamento.

$$
\tau=\frac{V}{t}
$$

Já para análises de DTR real de um fluido, algumas técnicas experimentais podem ser aplicadas. Entre elas, é utilizado o método de perturbação e resposta utilizando traçador não reativo. Este é injetado na linha de entrada do fluido a ser tratado, logo a montante da zona de contato sob concentração do traçador $\left(C_{t}\right)$ conhecida. A quantidade de traçador na saída da zona 


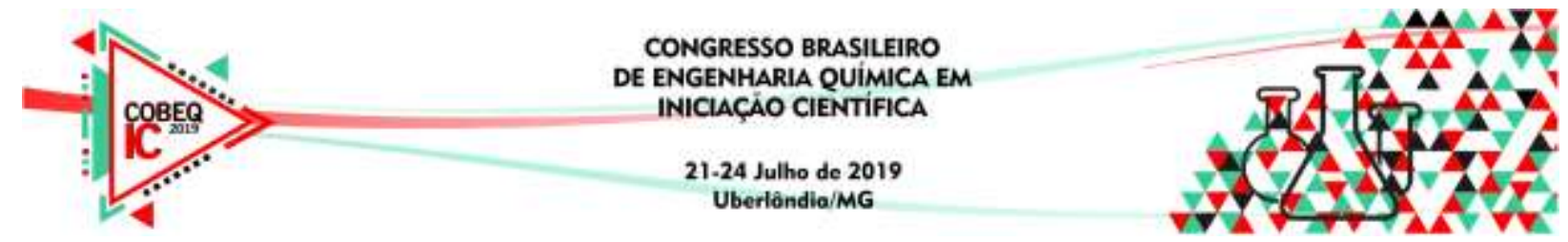

de contato é registrada continuamente ou em intervalos de tempo regulares (t). Desta forma, uma DTR do líquido pode ser obtida (SHAWWA e SMITH, 1998).

É conveniente que se represente a DTR de forma que a área sob a curva seja unitária (Equação (2)), ou seja, que os dados de concentrações coletadas sejam normalizados para determinar a área da curva acima do eixo do tempo (LEVENSPIEL, 2000).

$$
\int_{0}^{\infty} E(t) d t=1
$$

Onde $E(t)$ é a Distribuição do Tempo de Residência na saída e é calculado conforme Equação (3).

$$
E(t)=\frac{C(t)}{\int_{0}^{\infty} C(t) d t}
$$

onde $C(t)$ é a concentração do traçador em determinado tempo na saída da zona de contato.

O comportamento da Distribuição do Tempo de Residência pode se mostrar diferente dependendo do tipo de escoamento no sistema. A Figura 1 demonstra a característica da curva de concentração $\left(C_{(t / \bar{t})}\right)$ versus tempo relativo $(t / \bar{t})$ para sistemas (A) contínuos perfeitamente agitados; (B) sistemas não ideais; (C e D) tendendo a idealidade de uma perturbação de pulso ou pistão, respectivamente e (E) para sistemas tubulares com escoamento empistonado (NOVAIS, 2013).

Figura 1. Distribuições de tempos de residência para diversos tipos de sistemas.

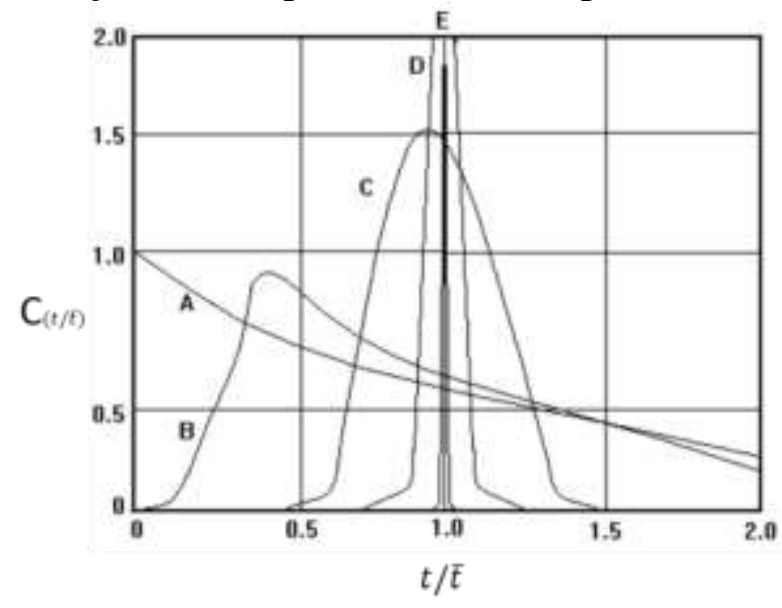

Fonte: NOVAIS, 2013 (Adaptado).

A partir do cálculo da Distribuição do Tempo de Residência pode-se determinar o tempo de residência médio caracterizado pela Equação (4). Este parâmetro é importante no conhecimento do tempo médio em que uma partícula permanece dentro do sistema, além de ser característica de escoamento não ideal. 


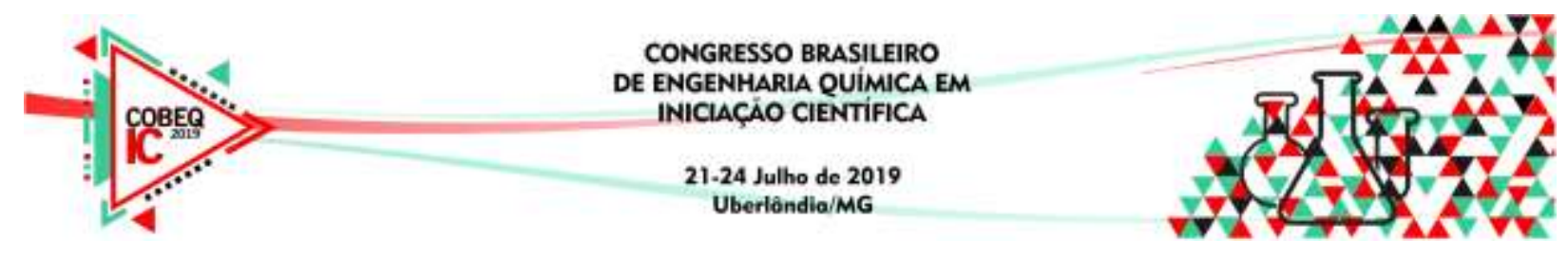

$$
t_{m}=\int_{0}^{\infty} t \cdot E(t) d t=\frac{\sum t_{i} C_{i} \Delta t_{i}}{\sum C_{i} \Delta t_{i}}
$$

De acordo com Levenspiel (2000), os modelos matemáticos podem representar escoamentos possibilitando comparar curvas obtidas experimentalmente às do modelo de escoamento ideal. Em flotadores nos quais curtos-circuitos, zonas mortas ou recirculação do fluido se manifestam, as curvas DTR experimentais caracterizam-se por apresentarem, respectivamente, picos antecipados em relação ao tempo de residência teórico, longas caudas e oscilações na curva (CESTARI et al., 2012).

Para um modelo de tanques em série, o número $(\mathrm{N})$ representa a quantidade de unidades através das quais o fluido escoa. O grau de mistura caracteriza-se pelo número de compartimentos. Desta forma, quanto maior o número de compartimentos, mais baixo é o grau de mistura. $\mathrm{O}$ delineamento das curvas ideais para este modelo é ilustrado na Figura 2, onde $\theta$ é a relação $t / t_{m}$.

Figura 2. Curvas de DTR para modelos de tanques em série.

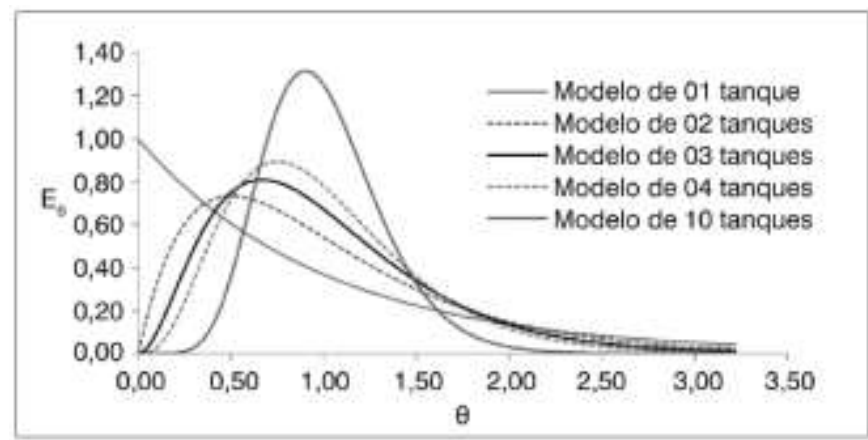

Fonte: Levenspiel, 2000 (Adaptado).

Ainda que cada unidade de flotação possua características específicas de escoamento, modelos matemáticos podem ser utilizados para estimar a quantidade de compartimentos (BRATBY, 2016) (Equação (5)).

$$
\tau=t_{m} \cdot \frac{N}{N-1}
$$

O objetivo deste trabalho foi averiguar o comportamento do escoamento de fluido entre os compartimentos de um flotador visando autentica-lo com modelos apresentados em literatura. O objetivo secundário foi determinar a Distribuição de Tempo de Residência nas etapas do processo de flotação representada por cada compartimento.

\section{METODOLOGIA EXPERIMENTAL}

\subsection{Distribuição do Tempo de Residência e tempo de residência médio}

Para determinação das DTR's foi utilizada a técnica de perturbação-resposta com o uso do traçador colorimétrico azul de metileno sob concentração de 1,0 g/L. Na unidade de flotação, 


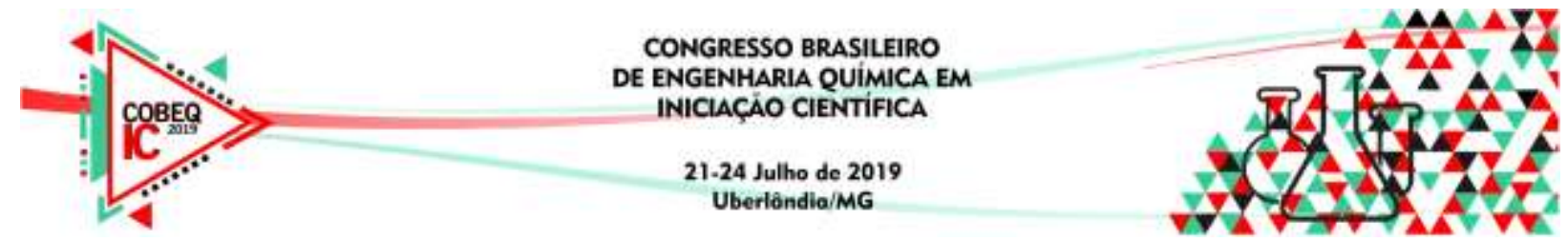

o tanque de efluente e o tanque de coagulante foram enchidos com água e, inicialmente, a vazão oferecida pela bomba peristáltica de efluente e de coagulante foram ajustadas à 4,70 e 0,23 $\mathrm{mL} / \mathrm{s}$ no painel, respectivamente. Desta forma, o tempo espacial total do processo foi pré-fixado conforme capacidade de funcionamento do saturador a uma pressão de 4,5 bar, sendo este um total de 20 minutos.

Foram produzidas DTR's específicas para três compartimentos distintos do equipamento: tanque de mistura $(1,00 \mathrm{~L})$, zona de contato $(1,10 \mathrm{~L})$ e zona de separação $(4,32 \mathrm{~L})$ com as tomadas de medidas nos pontos A, B e C (Figura 3). Todas as medidas foram realizadas com misturador a $170 \mathrm{rpm}$ no tanque de mistura.

Figura 3. Compartimentos estudados e pontos de coleta

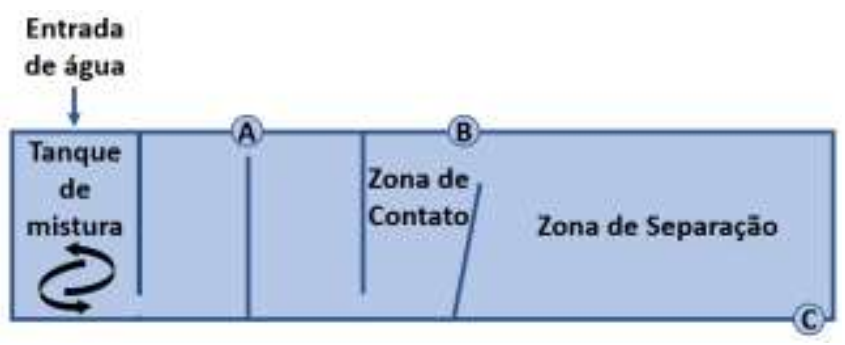

Fonte: Próprios autores.

Em cada determinação de DTR de cada compartimento o traçador azul de metileno foi injetado o mais rápido possível, para que se aproximasse de um pulso teórico instantâneo. As amostras foram recolhidas na saída de cada etapa em provetas e em tempos previamente determinados. Conveio as tomadas de medidas de 20 em 20 segundos até 1/3 do tempo total de análise para as zonas de contato e separação. Após isso, fez-se a coleta de $1 \mathrm{em} 1$ minuto. Isso porque há tendência de um escoamento maior de traçador no início do processo e a necessidade de medidas mais sensíveis. Para a etapa de mistura rápida as coletas foram fixadas em intervalos de 10 segundos. As leituras de absorbância do azul de metileno foram realizadas imediatamente no espectrofotômetro BEL PHOTONICS modelo SP 1105. O comprimento de onda escolhido foi de $665 \mathrm{~nm}$ que, segundo Vogel et al. (2002), corresponde a faixa espectral mais próxima do corante azul de metileno, exibindo o máximo de absorção seletiva e a máxima sensibilidade.

\section{RESULTADOS E DISCUSSÃO}

A Distribuição do Tempo de Residência para a etapa de mistura rápida é mostrada na Figura 3.1 (a). O comportamento da DTR se assemelhou ao modelo para um tanque (LEVENSPIEL, 2000) com efeito de curto-circuito representando uma baixa qualidade de mistura. O tempo médio experimental gerado foi de 83,40 segundos e, segundo Libânio (2010), infere-se que para maiores tempos de floculação, associados a gradientes de velocidade de menor magnitude, a eficiência na remoção das partículas tende a crescer, os flocos formados tendem a apresentar maiores dimensões e menor densidade, pois neles são incorporadas alta percentagem de água. A menor sedimentabilidade originada na etapa de floculação pode ser uma aliada no processo e aumentar a eficiência de coleta de partículas aglomeradas na etapa de contato com as microbolhas. 


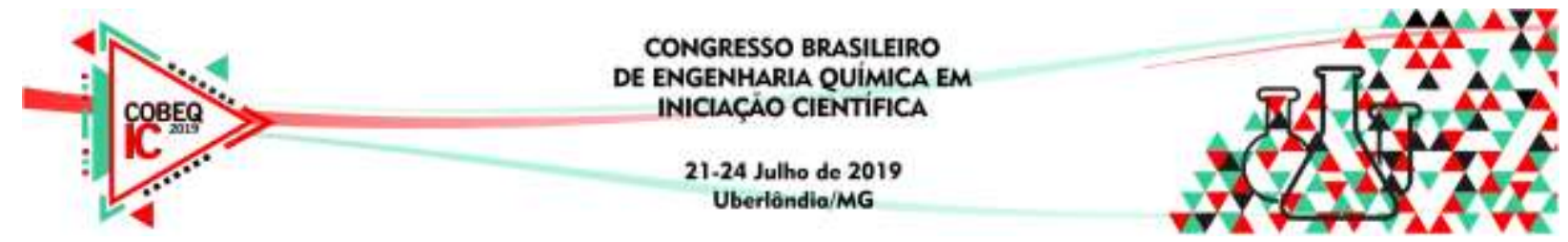

Em relação à DTR da zona de contato (Figura 4 (b)), o comportamento foi semelhante ao escoamento de fluido por 2 compartimentos em série, já que o tempo de detenção real $(118,70$ segundos) foi de aproximadamente 50\% do teórico (217,26 segundos), concordando com a Equação (5).

A partir das concentrações de traçador coletadas na saída do flotador (Figura 4 (c)), observou-se uma iminência de formação de pico aos 30 segundos e outra passagem bem formada aos 180 segundos, significando recirculação do fluido (LEVENSPIEL, 2000). Também houve oscilações na passagem de azul de metileno. Uma das justificativas é o comportamento pulsante do líquido proporcionado pela bomba peristáltica auxiliando que o escoamento formasse zonas de estagnação do traçador, além de presença de caminhos preferenciais e/ou indução de zonas mortas, significando um escoamento notavelmente não ideal (LEVENSPIEL, 2000).

Figura 4. Distribuições do Tempo de Residência nas etapas do flotador

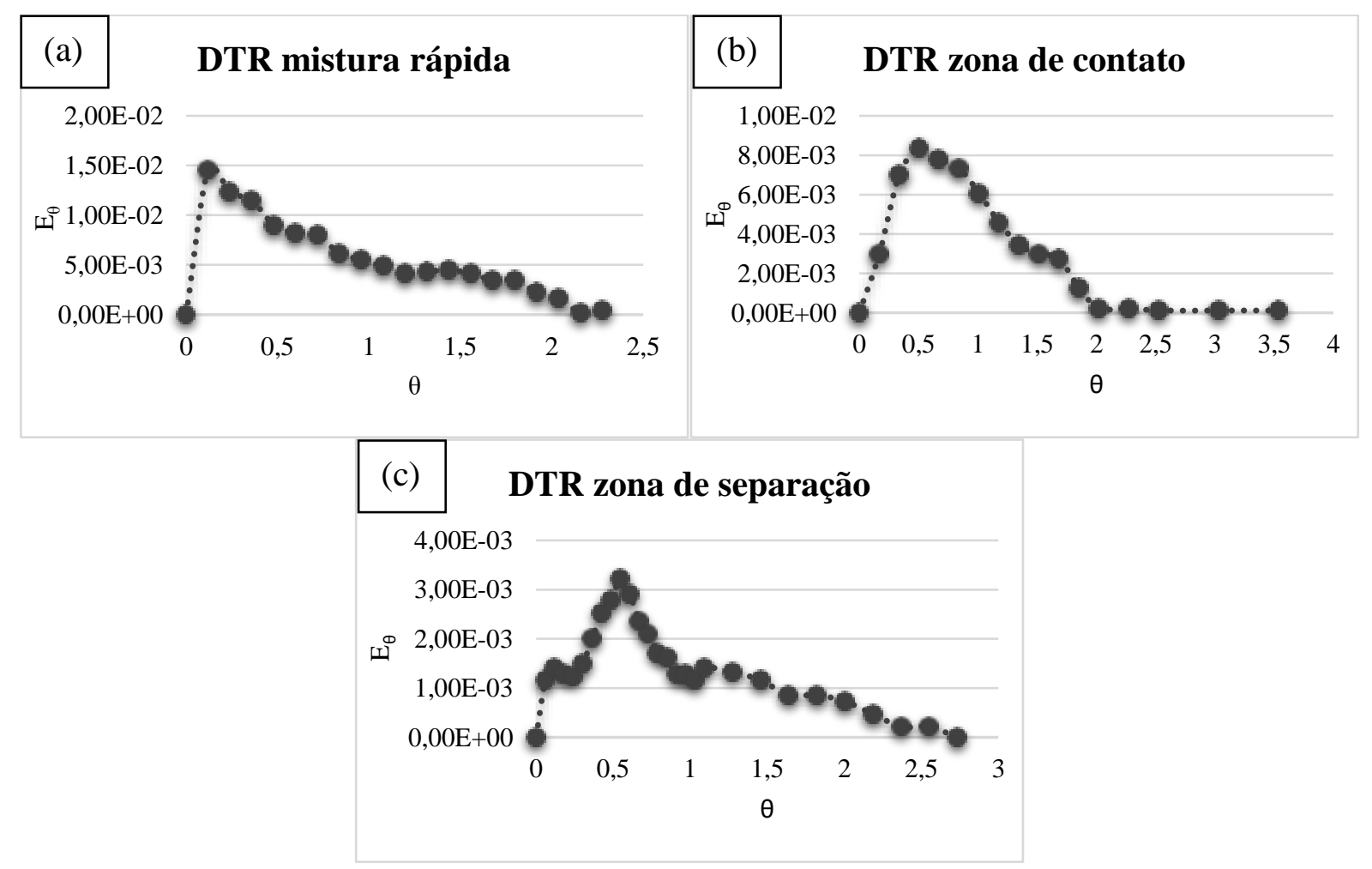

Fonte: Próprios autores.

\section{CONCLUSÃO}

As etapas estudadas no flotador em questão tiveram comportamentos segundo modelos da literatura. A etapa de mistura rápida apresentou efeito de curto-circuito (passagem antecipada de traçador) indicando baixa qualidade de mistura. Entretanto, houve alto tempo de residência médio o que pode aumentar a eficiência do processo. A zona de contato apresentou comportamento semelhante a dois tanques em série. Já na zona de separação, o escoamento 
exibiu levemente comportamento de recirculação. Por fim, conclui-se que as Distribuições de Tempo de Residência nas etapas do processo de flotação podem ser previsíveis conforme bibliografia analisada.

\section{REFERÊNCIAS}

BRATBY, J. Coagulation and flocculation in water and wastewater treatment. IWA Publishing, 2016.

CESTARI, J.L.; MATSUMOTO, T.; SOBRINHO, M.D.A.; LIBÂNIO, M. Avaliação hidrodinâmica de unidade piloto de floculação mecanizada. Revista Engenharia Sanitária Ambiental. V.17, nº1 p. 95-106, 2012.

LEVENSPIEL, O. Engenharia das Reações Químicas. 3 ed. São Paulo: Blucher, 2000.

LIBÂNIO, M. Fundamentos da Qualidade e Tratamento de Água. 3 ed. Campinas: Átomo, 2010.

NOVAIS, A.F. Experimento de reatores: batelada, CSTR, PFR. Departamento de Engenharia Química - UFSC. Florianópolis, 2013.

SHAWWA, A.R.; SMITH, D.W. Hydrodynamic Characterization in Dissolved Air Flotation (FAD) Contact Zone. Water Science and Technology. v.38, nº6 p. 245-252, 1998.

VOGEL, A.I; MENDHAM, J; DANNEY, R.C; BARNES, J.D. Análise química quantitativa. 6 ed. Rio de Janeiro: LTC, 2002. 